\title{
Retrospective Analysis of Nodal Spread Patterns According to Tumor Location in Pathological N2 Non-small Cell Lung Cancer
}

\author{
Yoshihisa Shimada $\cdot$ Hisashi Saji $\cdot$ Masatoshi Kakihana $\cdot$ \\ Hidetoshi Honda · Jitsuo Usuda • Naohiro Kajiwara • \\ Tatsuo Ohira $\cdot$ Norihiko Ikeda
}

Published online: 5 September 2012

(c) The Author(s) 2012. This article is published with open access at Springerlink.com

\begin{abstract}
Background The purpose of the present study was to determine the nodal spread patterns of $\mathrm{pN} 2$ non-small cell lung cancer (NSCLC) according to tumor location, and to attempt to evaluate the possible indications of selective lymph node dissection (SLND).

Methods We retrospectively analyzed nodal spread patterns in 207 patients with NSCLC of less than $5 \mathrm{~cm}$ with $\mathrm{N} 2$ involvement.

Results The tumor location was right upper lobe (RUL) in 79 , middle lobe in 12, right lower lobe (RLL) in 40, left upper division (LUD) in 41, lingular division in 11, and left lower lobe (LLL) in 24. Both RUL and LUD tumors showed a higher incidence of upper mediastinal (UM) involvement (96 and $100 \%$, respectively) and a lower incidence of subcarinal involvement (15 and $10 \%$, respectively) than lower lobe tumors (UM; RLL $60 \%$, LLL $42 \%$; subcarinal: RLL $60 \%$, LLL $46 \%$, respectively). Among the patients with 24 right UM-positive RLL and 10 left UM-positive LLL tumors, 2 showed negative hilar, subcarinal, and lower mediastinal involvement, and cT1, suggesting that UM dissection may be unnecessary in lower lobe tumors with no metastasis to hilar, subcarinal, and lower mediastinal nodes on frozen sections according to the preoperative $\mathrm{T}$ status. Among the patients with 12 subcarinal-positive RUL and 4 subcarinal-positive LUD tumors, one showed negative hilar or UM involvement, suggesting that subcarinal dissection may be unnecessary
\end{abstract}

Y. Shimada $(\bowtie) \cdot$ H. Saji $\cdot$ M. Kakihana $\cdot$ H. Honda

J. Usuda · N. Kajiwara · T. Ohira · N. Ikeda

First Department of Surgery, Tokyo Medical University

Hospital, 6-7-1 Nishishinjuku, Shinjuku-ku,

Tokyo 160-0023, Japan

e-mail: zenkyu@za3.so-net.ne.jp in RUL or LUD tumors with no metastasis to hilar and UM nodes on frozen sections.

Conclusions The present study appears to provide one of the supportive results regarding the treatment strategies for tumor location-specific SLND.

\section{Introduction}

Lobectomy with systematic mediastinal lymph node dissection (LND) has been considered the standard of care for resectable non-small cell lung cancer (NSCLC). Lymph node dissection was first reported by Cahan in 1960 [1] and is known to enhance staging accuracy by increasing lymph node harvesting and improving the identification of occult $\mathrm{N} 2$ disease. In contrast, other investigators claim that LND can potentially increased postoperative morbidity or may require longer operative time [2-5]. Some randomized controlled trials addressing the survival benefit of LND and mediastinal lymph node sampling showed no difference in survival outcome between patients undergoing LND and those undergoing lymph node sampling [3, 6, 7]. Whether or not patient outcome is improved by LND remains controversial.

At present, early lung cancers are more frequently encountered because of the widespread use of highresolution computed tomography $(\mathrm{CT})$ in routine practice and cancer screening $[8,9]$. Therefore, the extent of LND should be tailored to each patient. Selective lymph node dissection (SLND) based on the tumor location-specific lymphatic pathway should be undertaken especially for patients with no apparent lymph node metastasis or with impaired pulmonary function, or for elderly patients. In the present study, we retrospectively reviewed the prevalence of lymph node involvement in each mediastinal region in 
patients with N2 NSCLC according to the location of the primary tumor, and we attempted to evaluate the possible indications for SLND.

\section{Patients and methods}

Patients

From January 1990 to December 2007, a total of 2,195 patients underwent radical surgical resection of at least a lobectomy and systematic LND for NSCLC at our hospital. Of these 2,195 patients, we retrospectively analyzed lymph node spread patterns and outcome in 207 patients with NSCLC of less than $5 \mathrm{~cm}$ with $\mathrm{N} 2$ involvement. We excluded patients who had received preoperative treatment, including chemotherapy or chemoradiotherapy, those who had undergone only biopsy and SLND, and those who had low-grade malignant tumors. We also excluded patients with tumors spreading across lobar fissures and invading multiple lobes.

Preoperative evaluation included physical examination, chest radiography, computed tomography (CT) of the chest and abdomen, magnetic resonance imaging of the brain, bone scintigraphy, and blood examination. We determined that a large lymph node over $10 \mathrm{~mm}$ in the shortest axis was positive for metastasis on CT scans. Positron-emission tomography (PET) scan (recently integrated PET-CT scan) was not routinely used for staging resectable tumors during the study period. In recent years, endobronchial ultrasoundguided transbronchial needle aspiration (EBUS-TBNA) was sometimes performed for the patients having suspected multiple N2 lymph node metastases, but it was not routinely used. Similarly, mediastinoscopic biopsy was not routinely performed. Patients with N2 lymph node positively diagnosed by EBUS-TBNA or mediastinoscopic biopsy were excluded from the group of operative indication candidates.

The stage of disease was determined according to the 2009 7th Edition of the TNM Classification for Lung and Pleural Tumors [10]. The institutional review board of our institution approved the data collection and analyses and waived the need to obtain written informed consent from each patient.

\section{Operation}

During thoracotomy, lymph nodes in the ipsilateral thoracic cavity were completely resected. Systematic nodal dissection, including the superior to inferior mediastinum, was then performed after pulmonary resection. In cases of left thoracotomy, upper mediastinal dissection indicated aortic and tracheobronchial node dissection. If intraoperative findings showed that hilar or mediastinal lymph nodes were highly suspicious for metastatic disease, the resected lymph node specimens were immediately examined pathologically in frozen sections. Whether or not the presence or absence of lymph node metastasis was judged by intraoperative diagnosis, systematic LND was performed in the present study patients. Mediastinal metastases were considered to be skip metastases if any of the $\mathrm{N} 2$ nodes, but not the $\mathrm{N} 1$ nodes, were involved.

Mediastinal lymph node stations were grouped into the "zones" proposed by the International Association for the Study of Lung Cancer (IASLC) lung cancer staging project [11]. We also reviewed the correlation between nodal zone spread pattern and tumor location. We classified lymph node stations into the following six zones: the right upper (RU) and left upper (LU) zones, each including \#2R, \#3a, \#3p, and \#4R nodes; the subcarinal (SC) zone, including \#7 nodes; the right lower (RL) and left lower (LL) zones, each including \#8 and \#9 nodes; and the aortic-pulmonary (AP) zone, including \#5, and \#6 nodes.

Statistical analysis

Overall survival time was measured from the date of surgery to the date of death from any cause or the date on which the patient was last known to be alive. Survival curves were plotted according to the Kaplan-Meier method and compared with the log-rank test. Two-category comparison was performed by the Pearson $\chi^{2}$ test and Fisher's exact test for quantitative data. All tests were two-sided, and $p$ values of $<0.05$ were considered to indicate statistically significant differences. We used StatView 5.0 (SAS Institute Inc., Cary, NC) for the statistical analysis.

\section{Results}

Patient characteristics are summarized in Table 1. Of the 207 patients with NSCLC of less than $5 \mathrm{~cm}$ with N2 involvement, 55 (27\%) had skip metastasis, and 97 (47\%) had both hilar and the remaining 55 patients had metastatic segmental lymph nodes or subsegmenta lymph nodes with mediastinal lymph nodes metastasis. In addition, 74 (36\%) were diagnosed with $\mathrm{cN} 2$ disease by the chest CT. Lymph node spread patterns according to primary tumor location are presented in Fig. 1. The most common site of involvement for tumors of the right upper lobe (RUL; $n=79$ ) was the RU zone ( $n=76$; Fig. 1a). Right upper lobe tumors showed a significantly higher incidence of RU zone metastasis than right lower lobe (RLL) tumors (96 vs. $60 \%$, $p<0.001$ : Fig. 1a, b). In contrast, when RU zone metastasis was present, RLL tumors showed a significantly higher incidence of simultaneous metastasis to the SC or RL zone 
Table 1 Patient characteristics $(n=207)$

\begin{tabular}{|c|c|c|}
\hline & $n$ & $(\%)$ \\
\hline Overall & 207 & $(100)$ \\
\hline \multicolumn{3}{|l|}{ Sex } \\
\hline Male & 134 & $(65)$ \\
\hline Female & 73 & $(35)$ \\
\hline \multicolumn{3}{|l|}{ Histologic type } \\
\hline Adenocarcinoma & 149 & $(72)$ \\
\hline Squamous cell carcinoma & 41 & $(20)$ \\
\hline Others & 17 & $(8)$ \\
\hline \multicolumn{3}{|l|}{ Tumor size $(\mathrm{cm})$} \\
\hline 2.0 & 38 & (18) \\
\hline $2.1-3.0$ & 55 & $(27)$ \\
\hline $3.1-5.0$ & 114 & $(55)$ \\
\hline \multicolumn{3}{|l|}{$\mathrm{p}-\mathrm{T}$ status } \\
\hline pT1 & 47 & $(23)$ \\
\hline pT2 & 129 & $(62)$ \\
\hline pT3 & 18 & (9) \\
\hline pT4 & 13 & (6) \\
\hline \multicolumn{3}{|l|}{ Hilar lymph node metastasis } \\
\hline Present & 97 & $(47)$ \\
\hline Absent & 110 & $(53)$ \\
\hline \multicolumn{3}{|l|}{ Skip metastasis } \\
\hline Present & 55 & $(27)$ \\
\hline Absent & 152 & $(73)$ \\
\hline \multicolumn{3}{|l|}{ Tumor location } \\
\hline Right upper lobe & 79 & $(38)$ \\
\hline Right middle lobe & 12 & (6) \\
\hline Right lower lobe & 40 & (19) \\
\hline Left upper division & 41 & $(20)$ \\
\hline Left lingular division & 11 & (5) \\
\hline Left lower lobe & 24 & $(12)$ \\
\hline \multicolumn{3}{|l|}{ Procedure } \\
\hline Pneumonectomy & 15 & (7) \\
\hline Bilobectomy & 19 & (9) \\
\hline Lobectomy & 173 & $(84)$ \\
\hline
\end{tabular}

than RUL tumors ( 28 vs. $11 \%, p=0.026$ : Fig. $1 \mathrm{a}, \mathrm{b}$ ). The incidence of skip metastasis to only the RU zone was statistically lower among patients with RLL tumors than among those with RUL tumors ( 8 vs. $30 \%, p=0.005$ : Fig. 1a, b). Right upper lobe tumors showed a significantly lower incidence of SC zone metastasis than RLL tumors (15 vs. $60 \%, p<0.001$ : Fig. 1c, d). Most RUL tumors with SC zone metastasis showed simultaneous metastasis to the RU zone or hilar lymph nodes, and only one patient showed skip metastasis to the SC zone (Fig. 1c).

The most common site of involvement for tumors of the left upper division (LUD) $(n=41)$ was the AP or LU zone ( $n=41 ; 100 \%$ : Fig. 1e). Left upper division tumors showed a significantly higher incidence of AP or LU zone metastasis than left lower lobe (LLL) tumors (100 vs. $42 \%, p<0.001$ : Fig. 1e, g). In contrast, when AP or LU zone metastasis was present, LLL tumors showed a higher incidence of simultaneous metastasis to the SC or LL zone than LUD tumors, but the difference was not significant (29 vs. $12 \%, p=0.089$ : Fig. $1 \mathrm{e}, \mathrm{g})$. The incidence of skip metastasis to only the AP or LU zone was $45 \%$ in left lingular division tumors, $20 \%$ in LUD tumors, and $0 \%$ in LLL tumors, but the difference was not significant (Fig. 1e-g). Left upper division tumors showed a significantly lower incidence of SC zone metastasis than LLL tumors (10 vs. $46 \%, p<0.001$ : Fig. $1 \mathrm{~h}, \mathrm{j}$ ). All LUD tumors with SC zone metastasis showed simultaneous metastasis to the AP or LU zone, but no patient showed skip metastasis to the SC zone (Fig. 1h).

Patients were further categorized as those with tumors of the lower lobes ( $n=64 ; 40$ of right and 24 of left) and those with RUL or LUD tumors $(n=120 ; 79$ of RUL and 41 of LUD). The prognosis of patients with lower lobe tumors and RUL or LUD tumors was analyzed. The 5-year overall survival (OS) rates of patients with tumors of the lower lobes with upper mediastinal metastasis $(n=34$, $22 \%$ ) were poorer than, but not significantly different from, those of the patients without upper mediastinal metastasis $(n=30,34 \%)(p=0.371$; Fig. 2$)$. The 5-year OS rates of patients with RUL or LUD tumors with SC zone metastasis $(n=16,14 \%)$ were poorer than, but not significantly different from, those of the patients without SC zone metastasis $(n=104,40 \%)(p=0.073$; Fig. 3$)$.

The combined treatment strategies for tumor locationspecific SLND in N2 NSCLC patients according to clinical $\mathrm{T}$ status are summarized in Table 2. Among 24 patients with upper mediastinal metastasis from RLL tumors, nine showed no evidence of hilar, SC zone, and lower mediastinal metastasis. Of these nine patients, only one had clinical T1. Similarly, among ten patients with upper mediastinal metastasis from LLL tumors, only one showed no evidence of hilar, SC zone, and lower mediastinal metastasis, and clinical T1 status. Upper mediastinal dissection may be unnecessary in lower lobe tumors with negative hilar, SC and lower mediastinal nodes on frozen sections if the preoperative $\mathrm{T}$ status is $\mathrm{T} 1$ (Table 3). In contrast, among 12 patients with SC zone metastasis from RUL tumors, one showed no evidence of hilar or RU zone metastasis, and that tumor was classified as clinical T2. Among four patients with SC zone metastasis from LUD tumors, none showed evidence of hilar, upper mediastinal metastasis. This finding supports the hypothesis that SC dissection may be unnecessary in RUL and LUD tumors with no metastasis to hilar and upper mediastinal nodes on frozen sections, regardless of the clinical T status. Figure 4 shows diagrams of the main pathways of lymphatic spread of tumors according to tumor location. 
(a)

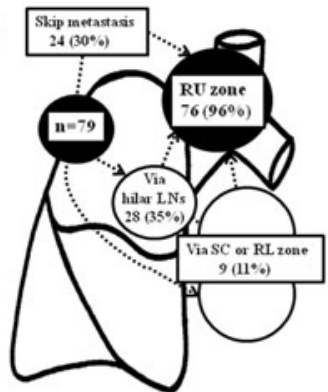

(e)

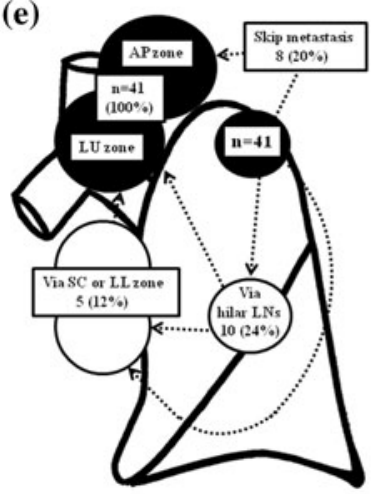

(i)

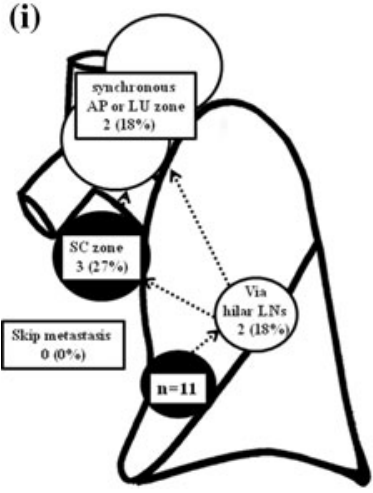

(b)

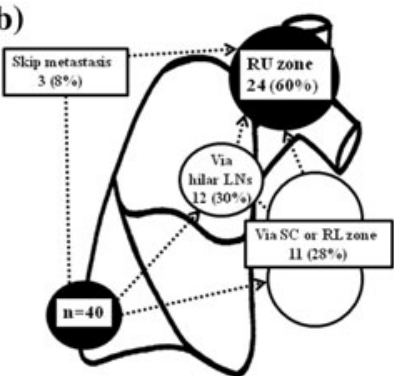

(f)

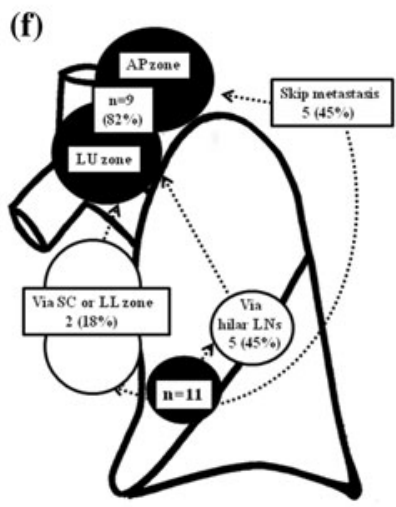

(j)

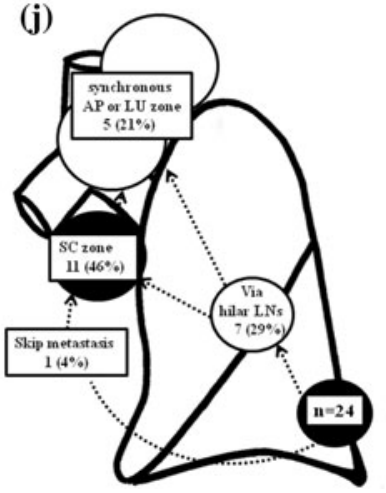

Fig. 1 Lymph node spread patterns according to the primary tumor location: a tumors of the right upper lobe (RUL) and right upper mediastinal metastasis. b Tumors of the right lower lobe (RLL) and right upper mediastinal metastasis. c Tumors of RUL and subcarinal metastasis. d Tumors of RLL and subcarinal metastasis. e Tumors of the left upper division (LUD) and left upper mediastinal metastasis.

\section{Discussion}

We set out to gain insight into the prevalence of lymph node metastasis in each mediastinal region in patients with $\mathrm{pN} 2$ NSCLC. The lymphatic pathways by which metastases from primary tumors in various segments and lobes spread toward the hilar and mediastinal lymph nodes have been investigated for over 50 years [12]. Studies of the patterns of location-specific lymphatic pathways of the lung have led to a better understanding of the importance of lymph node staging in the management of lung cancers. Although systematic LND consistently yields precise staging information,
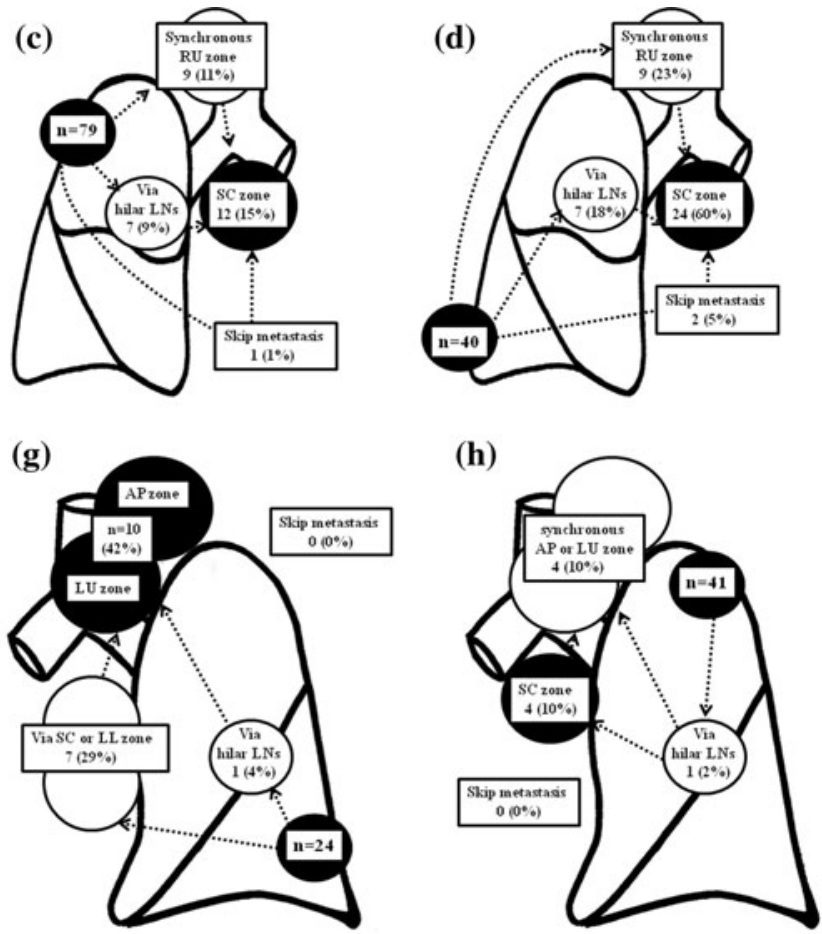

f Tumors of the left lingular division (LLD) and left upper mediastinal metastasis. g Tumors of the left lower lobe (LLL) and left upper mediastinal metastasis. $\mathbf{h}$ Tumors of LUD and subcarinal metastasis. i Tumors of LLD and subcarinal metastasis. $\mathbf{j}$ Tumors of LLL and subcarinal metastasis

it may contradict the concept of the optimal extent of lymph node dissection based on the location of the tumor. Some authors have postulated that the dissection of lymph nodes without cancer cells causes higher morbidity and mortality because it extends the operative procedure [2,6]. Moreover, the significance of LND regarding long-term outcome is still controversial. We therefore retrospectively reviewed the prevalence of mediastinal lymph node involvement in 207 patients with NSCLC of less than $5 \mathrm{~cm}$ with N2 involvement based on the location of the primary tumor, and we set out to determine the possible indications of location-specific SLND. 


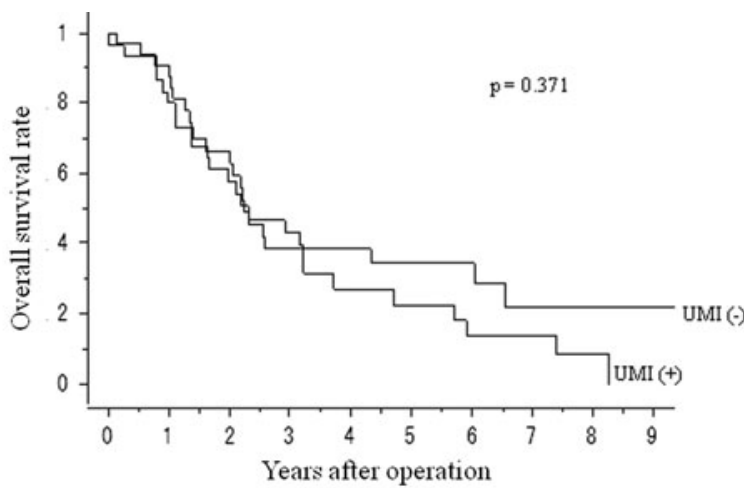

Patientsatrisk

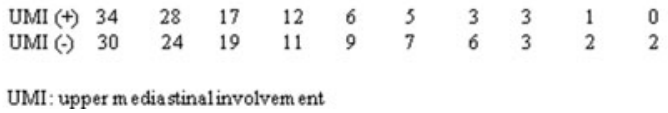

Fig. 2 Overall survival curves of lower lobe non-small cell lung cancer (NSCLC) pN2 patients, with or without upper mediastinal metastasis

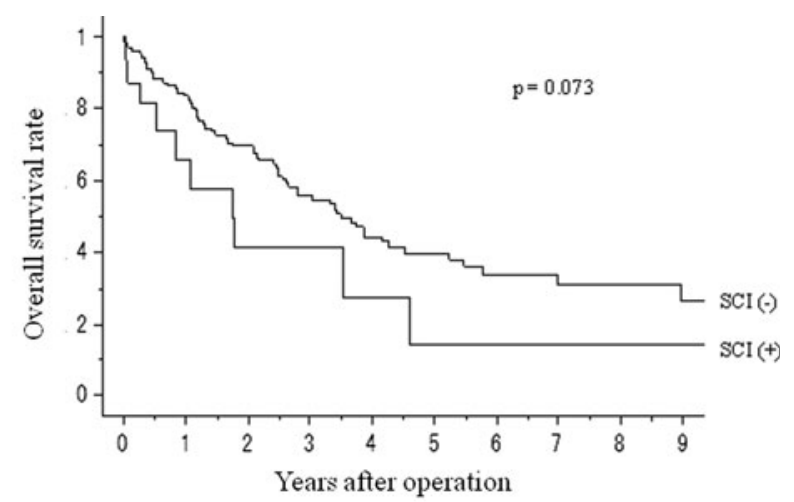

Patients atrisk

$$
\begin{array}{lllllllllll}
\text { SCI (+) } & 16 & 8 & 4 & 3 & 2 & 1 & 1 & 1 & 1 & 1 \\
\text { SCI (-) } & 104 & 84 & 70 & 45 & 32 & 24 & 15 & 9 & 8 & 6 \\
\\
\text { SCI: subcarinal involvem ent }
\end{array}
$$

Fig. 3 Overall survival curves of right upper lobe or left upper division NSCLC pN2 patients, with or without subcarinal metastasis

The IASLC staging project proposed the zone classification for future survival analyses [11]. Lee et al. [13] reported that grouping patients together according to zones provides accurate prognostic stratification for patients, and may resolve the ambiguity of the anatomical border, indicating applicability in the clinical setting. Therefore, we used the lymph node zone classification in this study.

Several retrospective studies have shown patterns of mediastinal lymph node metastases in relation to the location of the primary tumor [14-19]. Most of these studies have demonstrated that mediastinal lymph node metastases from RUL tumors occur predominantly in the $\mathrm{RU}$ area, but rarely in the SC area, whereas those from left
Table 2 Strategy for tumor location-specific selective nodal dissection in N2 non-small cell lung cancer (NSCLC) patients: distribution of upper mediastinal involvement according to clinical $\mathrm{T}$ status

Tumor location

\begin{tabular}{llllll}
\hline & RUL & RLL & LUD & LLD & LLL \\
\hline $\begin{array}{l}\text { No. of patients } \\
\text { with N2 }\end{array}$ & $79(100)$ & 40 & 41 & 11 & 24 \\
$\begin{array}{l}\text { No. of patients } \\
\text { with UMI }\end{array}$ & $76(96)$ & $24(60)$ & 41 & $9(82)$ & $10(42)$ \\
$\begin{array}{l}\text { Patients with } \\
\text { UMI }\end{array}$ & & & $(100)$ & & \\
$\begin{array}{l}\text { HI (-), SCI } \\
(-), \text { LMI (-) }\end{array}$ & $44(56)$ & $9(23)$ & $22(54)$ & $5(45)$ & $2(8)$ \\
Clinical T1 & $14(18)$ & $1(4)$ & $5(12)$ & $2(18)$ & $1(4)$ \\
Clinical T2-4 & $30(38)$ & $8(21)$ & $17(41)$ & $3(27)$ & $1(4)$ \\
\hline
\end{tabular}

$R U L$ right upper lobe, $R L L$ right lower lobe, $L U D$ left upper division, $L L D$ left lingular division, $L L L$ left lower lobe, $U M I$ upper mediastinal involvement, $H I$ hilar lymph node involvement, $S C I$ subcarinal involvement, $L M I$ lower mediastinal involvement

Table 3 Strategy for tumor location-specific selective nodal dissection in N2 NSCLC patients: distribution of subcarinal involvement

\begin{tabular}{|c|c|c|c|c|c|}
\hline \multicolumn{6}{|l|}{ Tumor location } \\
\hline & $\begin{array}{l}\text { RUL } \\
n(\%)\end{array}$ & RLL & LUD & LLD & LLL \\
\hline $\begin{array}{l}\text { No. of } \\
\text { patients } \\
\text { with N2 }\end{array}$ & 79 (100) & $40(100)$ & $41(100)$ & $11(100)$ & $24(100)$ \\
\hline $\begin{array}{l}\text { No. of } \\
\text { patients } \\
\text { with SCI }\end{array}$ & $12(15)$ & $24(60)$ & $4(10)$ & $3(27)$ & $11(46)$ \\
\hline \multicolumn{6}{|l|}{$\begin{array}{l}\text { Patients with } \\
\text { SCI }\end{array}$} \\
\hline $\begin{array}{l}\mathrm{HI}(-), \mathrm{UMI} \\
(-)\end{array}$ & $1(1)$ & $3(8)$ & $0(0)$ & $0(0)$ & $3(13)$ \\
\hline Clinical T1 & $0(0)$ & $1(3)$ & $0(0)$ & $0(0)$ & $0(0)$ \\
\hline Clinical T2-4 & $1(1)$ & $2(5)$ & $0(0)$ & $0(0)$ & $3(13)$ \\
\hline
\end{tabular}
according to clinical $\mathrm{T}$ status

upper lobe tumors occur most frequently in the AP or LU area, but those from tumors of the lower lobes rarely occur in the upper mediastinal area. In the present study, metastases to the SC zone from RUL or LUD tumors were significantly less frequent (15 and $12 \%$, respectively) than metastases to the SC zone from tumors of the lower lobes. The outcome of patients with RUL or LUD tumors with SC zone metastasis was poorer than, but not significantly different from, that of patients with RUL and LUD tumors without SC zone metastasis $(p=0.073)$. There was only 1 patient with only SC zone skip metastasis. Patients with upper lobe NSCLC involving SC nodes are reportedly rare $[16,18,19]$, and they have poorer outcomes than those 

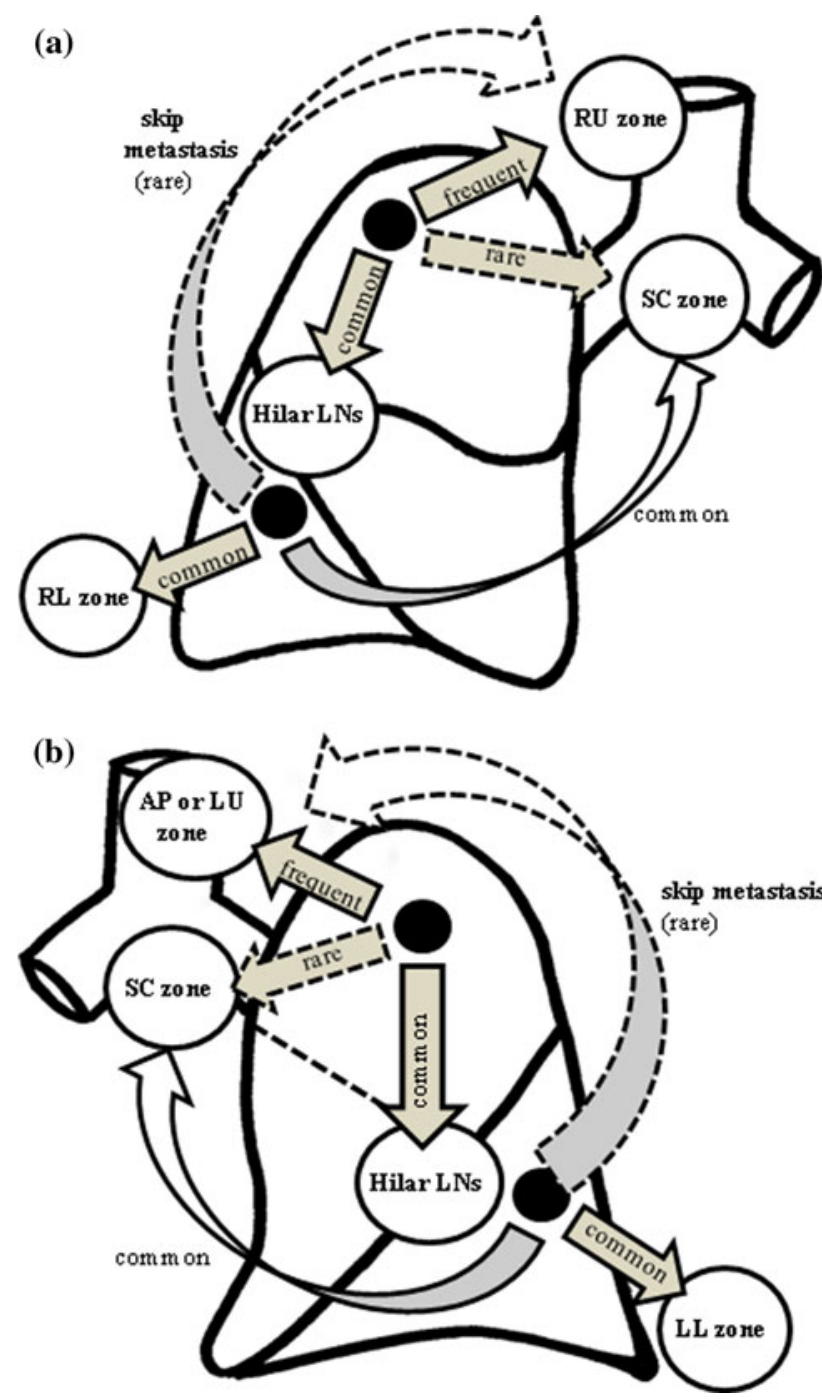

Fig. 4 Diagrams of the main pathways of lymphatic spread according to tumor location. a In right-side tumors, almost all RUL tumors metastasized to the RU zone directly or through the hilar lymph node. RUL tumors metastasized less frequently to the SC zone. Right lower lobe (RLL) tumors metastasized to various mediastinal lymph node zones, and skip metastasis to the RU zone was rare in RLL tumors. b In left-side tumors, all LUD tumors metastasized to the AP zone directly or through the hilar lymph node. Upper lobe tumors metastasized less frequently to the SC zone. Left lower lobe (LLL) tumors metastasized to various mediastinal lymph node zones, and skip metastasis to the AP zone was rare in LLL tumors

without SC node metastasis [19]. Based on these results, we also evaluated the possible indications of tumor location-specific SLND. Although we did not routinely perform frozen section diagnosis of sampled hilar lymph nodes, we conducted a frozen section examination intraoperatively if metastasis was suspected. There was only 1 patient with SC zone metastasis from RUL tumors who did not show any evidence of hilar and RU zone metastases, whereas no SC zone metastasis from any LUD tumors was observed when neither the hilar nor RU zone showed any evidence of metastasis. Resection of the SC zone in the case of RUL and LUD tumors may be unnecessary if neither upper mediastinal nor hilar lymph nodes show any evidence of metastasis on frozen sections, regardless of the clinical $\mathrm{T}$ status.

There were fewer patients with metastases to the upper mediastinal zone from tumors of the lower lobes than with metastases to the upper mediastinal zone from tumors of the upper lobes. The outcome of patients with tumors of the lower lobes with upper mediastinal metastasis was poorer than, but not significantly different from, that of patients with tumors of the lower lobes without upper mediastinal metastasis $(p=0.371)$. There was only one patient each with RU zone metastasis from a clinical T1 RLL tumor and AP zone metastasis from a clinical T1 LLL tumor, but neither showed any evidence of lymph node metastasis to the SC node, lower mediastinal zone, and hilum. Therefore resection of upper mediastinal zones in tumors of the lower lobes may be unnecessary even if the preoperative $\mathrm{T}$ status is T1, and if lymph node biopsies in the SC node, lower mediastinal zone, and hilum do not show any evidence of metastasis on frozen sections. However, former studies indicated that the superior and basal segment lung cancers in the lower lobe have different lymph node metastasis patterns [14]. Although there was no significant difference in the metastasis patterns of lower lobe tumors, this finding may be attributable to the small number of patients in the present study (data not shown). The strategy of lymph node dissection should be changed from extensive dissection to SLND, especially in early stage cancer or poor-risk patients, because SLND can reduce postoperative morbidity associated with such complications as bronchopleural fistula, chylothorax, or recurrent nerve palsy [2-5]. However, lung cancer can easily metastasize to the mediastinum, and therefore patient selection should be determined carefully. If patients are suspected of having advanced disease based on intraoperative findings, LND should be performed.

The present study has several limitations. It was a retrospective study, and possible bias may exist. First, we examined suspected hilar or mediastinal lymph nodes intraoperatively in frozen sections, but specific systemic sampling methodologies have been established and used in the past. Second, the number of patients in this study may be too small to draw any definitive conclusion. Third, current less-invasive staging modalities, including PET-CT or EBUS were infrequently used because of the inclusion of a large amount of data from old cases, collected at a time when these procedures were less well established. Thus we might have inadvertently performed some operations on undetected $\mathrm{N} 3$ disease.

In conclusion, we demonstrated the potential validity of refraining from resecting lymph nodes in the SC zone in 
cases of RUL or LUD tumors, or those in the upper mediastinal zone in the case of tumors of the lower lobes. Considering the fact that NSCLC patients can benefit from SLND, a prospective study is essential to confirm the effect of tumor location-specific SLND on survival and optimal postoperative treatment.

Acknowledgments The authors are grateful to Mr. Roderick J. Turner, Assistant Professor Edward F. Barroga, and Professor J. Patrick Barron, Chairman of the Department of International Medical Communications of Tokyo Medical University, for their editorial review of the English manuscript.

Open Access This article is distributed under the terms of the Creative Commons Attribution License which permits any use, distribution, and reproduction in any medium, provided the original author(s) and the source are credited.

\section{References}

1. Cahan WG (1960) Radical lobectomy. J Thorac Cardiovasc Surg 39:555-572

2. Izbicki JR, Thetter O, Habekost M et al (1994) Radical systematic mediastinal lymphadenectomy in non-small cell lung cancer: a randomized controlled trial. Br J Surg 81:229-235

3. Izbicki JR, Passlick B, Pantel K et al (1998) Effectiveness of radical systematic mediastinal lymphadenectomy in patients with resectable non-small cell lung cancer: results of a prospective randomized trial. Ann Surg 227:138-144

4. Lardinois D, Suter H, Hakki H et al (2005) Morbidity, survival, and site of recurrence after mediastinal lymph-node dissection versus systematic sampling after complete resection for nonsmall cell lung cancer. Ann Thorac Surg 80:268-274 (discussion 274-265)

5. Okada M, Sakamoto T, Yuki T et al (2006) Selective mediastinal lymphadenectomy for clinico-surgical stage I non-small cell lung cancer. Ann Thorac Surg 81:1028-1032

6. Sugi K, Nawata K, Fujita N et al (1998) Systematic lymph node dissection for clinically diagnosed peripheral non-small-cell lung cancer less than $2 \mathrm{~cm}$ in diameter. World J Surg 22:290-294. doi: 10.1007/s002689900384 (discussion 294-295)

7. Darling GE, Allen MS, Decker PA et al (2011) Randomized trial of mediastinal lymph node sampling versus complete lymphadenectomy during pulmonary resection in the patient with N0 or N1 (less than hilar) non-small cell carcinoma: results of the American College of Surgery Oncology Group Z0030 Trial. J Thorac Cardiovasc Surg 141:662-670

8. Henschke CI, McCauley DI, Yankelevitz DF et al (1999) Early Lung Cancer Action Project: overall design and findings from baseline screening. Lancet 354:99-105

9. Sone S, Takashima S, Li F et al (1998) Mass screening for lung cancer with mobile spiral computed tomography scanner. Lancet 351:1242-1245

10. Sobin LH, Gospodarowicz MK, Wittekind C (eds) (2009) TNM classification of malignant tumors. UICC, International Union Against Cancer. Wiley, Weinheim

11. Rusch VW, Crowley J, Giroux DJ et al (2007) The IASLC Lung Cancer Staging Project: proposals for the revision of the $\mathrm{N}$ descriptors in the forthcoming seventh edition of the TNM classification for lung cancer. J Thorac Oncol 2:603-612

12. Nohl HC (1956) An investigation into the lymphatic and vascular spread of carcinoma of the bronchus. Thorax 11:172-185

13. Lee JG, Lee CY, Bae MK et al (2008) Validity of international association for the study of lung cancer proposals for the revision of $\mathrm{N}$ descriptors in lung cancer. J Thorac Oncol 3:1421-1426

14. Watanabe S, Suzuki K, Asamura H (2008) Superior and basal segment lung cancers in the lower lobe have different lymph node metastatic pathways and prognosis. Ann Thorac Surg 85:10261031

15. Watanabe $\mathrm{S}$, Asamura $\mathrm{H}$, Suzuki $\mathrm{K}$ et al (2005) The new strategy of selective nodal dissection for lung cancer based on segmentspecific patterns of nodal spread. Interact Cardiovasc Thorac Surg 4:106-109

16. Okada M, Tsubota N, Yoshimura M et al (1998) Proposal for reasonable mediastinal lymphadenectomy in bronchogenic carcinomas: role of subcarinal nodes in selective dissection. J Thorac Cardiovasc Surg 116:949-953

17. Ishiguro F, Matsuo K, Fukui T et al (2010) Effect of selective lymph node dissection based on patterns of lobe-specific lymph node metastases on patient outcome in patients with resectable non-small cell lung cancer: a large-scale retrospective cohort study applying a propensity score. J Thorac Cardiovasc Surg 139:1001-1006

18. Asamura H, Nakayama H, Kondo H et al (1999) Lobe-specific extent of systematic lymph node dissection for non-small cell lung carcinomas according to a retrospective study of metastasis and prognosis. J Thorac Cardiovasc Surg 117:102-1111

19. Aokage K, Yoshida J, Ishii G et al (2010) Subcarinal lymph node in upper lobe non-small cell lung cancer patients: is selective lymph node dissection valid? Lung Cancer 70:163-167 